\title{
VEM AND TOPOLOGY OPTIMIZATION ON POLYGONAL MESHES
}

\author{
P.F. Antonietti ${ }^{1}$, M. Bruggi ${ }^{2}$, S. Scacchi ${ }^{3}$ and M. Verani ${ }^{1}$ \\ ${ }^{1}$ MOX-Department of Mathematics \\ Politecnico di Milano \\ Piazza Leonardo da Vinci, 3220133 Milano, Italy \\ e-mail: paola.antonietti@polimi.it,marco.verani@polimi.it \\ ${ }^{2}$ Department of Civil and Environmental Engineering DICA \\ Politecnico di Milano \\ Piazza Leonardo da Vinci, 3220133 Milano, Italy \\ e-mail: matteo.bruggi@polimi.it \\ ${ }^{3}$ Department of Mathematics \\ University of Milan \\ Via Saldini, 5020133 Milano, Italy \\ e-mail: simone.scacchi@unimi.it
}

Keywords: Topology Optimization, Virtual Element Method

\begin{abstract}
Topology optimization is a fertile area of research that is mainly concerned with the automatic generation of optimal layouts to solve design problems in Engineering. The classical formulation addresses the problem of finding the best distribution of an isotropic material that minimizes the work of the external loads at equilibrium, while respecting a constraint on the assigned amount of volume. This is the so-called minimum compliance formulation that can be conveniently employed to achieve stiff truss-like layout within a two-dimensional domain. A classical implementation resorts to the adoption of four node displacement-based finite elements that are coupled with an elementwise discretization of the (unknown) density field. When regular meshes made of square elements are used, well-known numerical instabilities arise, see in particular the so-called checkerboard patterns. On the other hand, when unstructured meshes are needed to cope with geometry of any shape, additional instabilities can steer the optimizer towards local minima instead of the expected global one. Unstructured meshes approximate the strain energy of truss-like members with an accuracy that is strictly related to the geometrical features of the discretization, thus remarkably affecting the achieved layouts. In this paper we will consider several benchmarks of truss design and explore the performance of the recently proposed technique known as the Virtual Element Method (VEM) in driving the topology optimization procedure. In particular, we will show how the capability of VEM of efficiently approximating elasticity equations on very general polygonal meshes can contribute to overcome the aforementioned mesh-dependent instabilities exhibited by classical finite element based discretization techniques.
\end{abstract}




\section{INTRODUCTION}

Topology optimization is a design tool that is extensively exploited in problems related to Engineering and its industrial applications. The classical formulation searches for the best distribution of a prescribed amount of isotropic material that minimizes the so-called compliance, e.g., twice the work of the external loads at equilibrium [7]. This framework is based on the adoption of a suitable penalization of the mechanical properties of the elastic body depending on the local values of the density field, see e.g. the well-known SIMP (Solid Isotropic Material with Penalization) [8]. A classical approach to the solution of a topology optimization problem resorts to the discretization of the density field to cope with the minimization setting, along with the adoption of finite element methods to tackle the approximation of the equilibrium equation. Methods of mathematical programming are adopted to solve the arising minimization problem, see [24]. In general, low-order displacement-based finite elements are coupled with an element-wise density discretization, being affected by numerical instabilities as checkerboard and mesh dependence. The former drawback depends on the choice of the displacement shape functions with respect to the adopted density approximation, whereas the latter is due to an inherent ill-posedness of the continuous problem [22]. Checkerboard and mesh dependence are solved, in general, via ad hoc filtering procedures that preserve the advantages of the above mentioned discrete approach. However, this approach suffers from drawbacks that are peculiar to the adopted low-order discretization of the state equation. It is worth mentioning, among the others, a lack of accuracy in the approximation of the displacement and stress fields and the arising of undesired locking phenomena when considering incompressible media. Variations of the basic approach have been investigated to solve the first issue, see among the others [15, 25] focusing on adaptivity. Robust discretizations to cope with incompressible materials have also been addressed, see the u-p formulation [23] and mixed finite elements [14]. Moreover, when unstructured meshes are needed to deal with geometry of any shape, additional instabilities can steer the optimizer towards local minima, instead of the expected global one, when using the classical approach. Unstructured meshes approximate the strain energy of optimal truss-like members with an accuracy that is strictly related to the geometrical features of the approximation, thus remarkably affecting the achieved layouts.

The aim of this paper is to investigate whether the recently proposed Virtual Element Method (VEM), cf. [3], can be effectively employed for topology optimization problems. Following the pioneering work in [17], several benchmarks of truss design will be investigated to explore the performance the Virtual Element Method when employed to drive the topology optimization algorithm. More precisely, we will demonstrate that the inherited flexibility of VEM to deal with general polygonal meshes can contribute to overcome the mesh-dependent instabilities featured by classical discretization techniques.

The Virtual Element Method (see, e.g., [3] for an introduction to the method and [4] for the details of its practical implementation) is characterized by the capability of dealing with very general polygonal/polyedral meshes and by the possibility of easily implementing highly regular discrete spaces. Indeed, by avoiding the explicit construction of the local basis functions, the VEM can easily handle general polygons/polyhedrons without complex integrations on the element. In addition, thanks to this added flexibility, it was discovered [12, 6] that virtual elements can also be used to build global discrete spaces of arbitrary regularity ( $C^{1}$ and more) that are quite simple in terms of degrees of freedom and coding. So far, VEM has been applied to 
a variety of different problems [11, 1, 5, 9, 10, 16, 18, 20, 21, 2]. However, apart from [17] a systematic study of the impact of VEM on topology optimization problems is still in its infancy. The aim of this paper is to contribute in filling this gap.

The outline of the paper is as follows. Section 2.1 recalls the classical topology optimization problem, whereas Section 2.2 provides fundamentals of the Virtual Element Method and introduces the VEM discrete volume-constrained minimum compliance problem. Section 2.3 addresses numerical instabilities, i.e. checkerboard and mesh dependence. Section 3 presents numerical simulations performed on benchmark examples that are based on the adoption of structured and unstructured meshes of both polygonal and quadrangular elements. Section 4 provides final remarks and outlines the ongoing research.

\section{GOVERNING EQUATIONS}

\subsection{Continuous problem}

Topology optimization for the maximization of the structural stiffness is herein addressed, according to the well-known minimum compliance formulation. The problem of distributing a given amount of linear elastic isotropic material is solved, such that the work of the external load against the corresponding displacement at equilibrium is minimized.

Let $\Omega \subset \mathbb{R}^{2}$ be an open, bounded domain of $\mathbb{R}^{2}$ with Lipschitz boundary $\Gamma=\partial \Omega$. Let $\rho \in \mathcal{Q}_{a d}=\left\{\rho \in L^{\infty}(\Omega): \quad 0<\rho_{\min } \leq \rho \leq 1\right.$ a.e. in $\left.\Omega\right\}$ be a bounded function representing the material density in $\Omega$, where $\rho_{\min }$ is some positive lower bound that is already introduced to avoid any singularity in the analysis of the further derived discrete problem. Let $\mathbf{C}=\mathbf{C}(\rho(x))$ be a fourth order elasticity tensor depending on the material density at the point $x \in \Omega$. According to the well-known SIMP model, see e.g. [8], we assume

$$
\mathbf{C}(\rho(x))=\rho(x)^{p} \mathbf{C}^{0} \quad p>1,
$$

where

$$
C_{i j h k}^{0}=\frac{E \nu}{(1+\nu)(1-2 \nu)} \delta_{i j} \delta_{h k}+\frac{E}{2(1+\nu)}\left(\delta_{i h} \delta_{j k}+\delta_{i k} \delta_{j h}\right)
$$

is the stiffness tensor for an assigned isotropic medium with Young modulus $E$ and Poisson's ratio $\nu$, whereas $p$ is a penalization parameter that is usually assumed to be equal to 3, see e.g. [7].

Let $\mathbf{u}_{d}$ and $\mathbf{f}_{t}$ denote the displacement and traction prescribed on $\Gamma_{d} \subset \Gamma$ and $\Gamma_{t}=\Gamma \backslash \Gamma_{d}$, respectively. For a given density material distribution $\rho$, the displacement field $\mathbf{u}$ solves the following state problem

$$
\begin{aligned}
\operatorname{div} \sigma+\mathbf{g} & =\mathbf{0} & & \text { in } \Omega \\
\sigma & =\mathbf{C}(\rho) \epsilon(\mathbf{u}) & & \\
\mathbf{u} & =\mathbf{u}_{d} & & \text { on } \Gamma_{d} \\
\sigma \cdot \mathbf{n} & =\mathbf{f}_{t} & & \text { on } \Gamma_{t},
\end{aligned}
$$

where $\epsilon(\mathbf{u})=\frac{1}{2}\left(\nabla \mathbf{u}+\nabla^{t} \mathbf{u}\right) \equiv \nabla^{s} \mathbf{u}$. According to a frequent assumption in topology optimization, no body load will be considered in the sequel, meaning that $\mathrm{g}=\mathbf{0}$. 
Let us introduce the semi-linear form $a(\rho ; \cdot, \cdot): \mathcal{Q}_{a d} \times\left[H^{1}(\Omega)\right]^{2} \times\left[H^{1}(\Omega)\right]^{2} \rightarrow \mathbb{R}$

$$
a(\rho ; \mathbf{u}, \mathbf{v}):=\int_{\boldsymbol{\Omega}} \mathbf{C}(\rho) \epsilon(\mathbf{u}): \epsilon(\mathbf{v}) \mathbf{d} \mathbf{x}
$$

and the linear functional $\mathcal{F}(\cdot):\left[H^{1}(\Omega)\right]^{2} \rightarrow \mathbb{R}$

$$
\mathcal{F}(\mathbf{v})=\int_{\Gamma_{\mathbf{t}}} \mathbf{f}_{\mathbf{t}} \cdot \mathbf{v} \mathbf{d x}
$$

We introduce the following spaces

$$
\begin{array}{ll}
\mathcal{V}_{0}=\left\{\mathbf{u} \in\left[H^{1}(\Omega)\right]^{2}:\right. & \left.\mathbf{u}=\mathbf{0} \text { on } \Gamma_{d}\right\} \\
\mathcal{V}=\left\{\mathbf{u} \in\left[H^{1}(\Omega)\right]^{2}:\right. & \left.\mathbf{u}=\mathbf{u}_{\mathbf{d}} \text { on } \Gamma_{d}\right\} .
\end{array}
$$

Then the weak formulation of the problem (2a)-(2d) reads as follows: for a given $\rho \in \mathcal{Q}_{a d}$ find $\mathbf{u} \in \mathcal{V}$ such that

$$
a(\rho ; \mathbf{u}, \mathbf{v})=\mathcal{F}(\mathbf{v})
$$

for all $\mathbf{v} \in \mathcal{V}_{0}$

According to the Clapeyron theorem, the continuous formulation of the topology optimization problem for minimum compliance may be therefore written as:

$$
\begin{cases}\min _{\rho \in \mathcal{Q}_{a d}} & \mathcal{C}(\rho, \mathbf{u})=\int_{\Gamma_{t}} \mathbf{f}_{t} \cdot \mathbf{u} d x=\int_{\Omega} \rho^{p} \mathbf{C}_{0} \epsilon(\mathbf{u}): \epsilon(\mathbf{u}) d x \\ \text { s.t. } & a(\rho ; \mathbf{u}, \mathbf{v})=\mathcal{F}(\mathbf{v}) \forall \mathbf{v} \in \mathcal{V}_{\mathbf{0}} \\ & \frac{1}{V} \int_{\Omega} \rho d x \leq V_{f},\end{cases}
$$

being $V_{f}$ the available amount of material as a fraction of the whole domain $V=\int_{\Omega} 1 d x$. Minimizing the compliance $\mathcal{C}$ of a structure acted upon by a prescribed set of assigned forces means minimizing the work of external loads, i.e. looking for a stiff structure.

\subsection{Virtual Element discretization}

In this section we introduce the Virtual Element discretization of problem (4). From now on, we will assume that $\Omega$ is a polygonal domain in $\mathbb{R}^{2}$. Let $\mathcal{T}_{h}$ represent a decomposition of $\Omega$ into general, possibly non-convex, polygonal elements $E$ with $\operatorname{diam}(E)=h_{E}$, where $\operatorname{diam}(E)=\max _{x, y \in E}\|x-y\|$. In the following, we will denote by $e$ the straight edges of the mesh $\mathcal{T}_{h}$ and, for all $e \in \partial E, \mathbf{n}_{E}^{e}$ will denote the unit normal vector to $e$ pointing outward to $E$. We will use the symbol $\mathbb{P}_{k}(\omega)$ to denote the space of polynomials of degree less than or equal to $k$ living on the set $\omega \subseteq \mathbb{R}^{2}$.

Let us first introduce the finite dimensional space of piecewise constant admissible controls

$$
\mathbb{Q}_{a d}=\left\{\rho_{h} \in \mathcal{Q}_{a d}: \rho_{h \mid E} \in \mathbb{P}_{0}(E) \forall E \in \mathcal{T}_{h}\right\} .
$$

Clearly, a function $\rho_{h} \in \mathbb{Q}_{a d}$ is uniquely determined by its value $\rho_{E}$ in each polygon $E \in \mathcal{T}_{h}$. Hence, the dimension of $\mathbb{Q}_{a d}$ equals the cardinality of $\mathcal{T}_{h}$. 
Moreover, following [5], it is possible to introduce the low-order discrete VEM spaces $\mathbf{V}_{0, h} \subset \mathcal{V}_{0}$ and $\mathbf{V}_{h} \subset \mathcal{V}$, a discrete form $a_{h}\left(\rho_{h} ; \mathbf{u}_{h}, \mathbf{v}_{h}\right)$ approximating $a(\rho ; \mathbf{u}, \mathbf{v})$ and a discrete functional $\mathcal{F}_{h}\left(\mathbf{v}_{h}\right)$ approximating $\mathcal{F}(\mathbf{v})$ such that the VEM discretization of (3) reads as: given $\rho_{h} \in \mathbb{Q}_{a d}$ find $\mathbf{u}_{h} \in \mathcal{V}_{h}$ such that

$$
a_{h}\left(\rho_{h} ; \mathbf{u}_{h}, \mathbf{v}_{\mathbf{h}}\right)=\mathcal{F}_{\mathbf{h}}\left(\mathbf{v}_{\mathbf{h}}\right)
$$

for any $\mathbf{v}_{\mathbf{h}} \in \mathcal{V}_{\mathbf{0}, \mathbf{h}}$. In particular, the global VEM spaces $\mathbf{V}_{0, h}$ and $\mathbf{V}_{h}$ are obtained by gluing suitable local discrete VEM spaces, denoted by $\mathcal{V}_{h}(E)$, whose elements are uniquely identified by the values at the vertices of the polygon $E$ and contain linear polynomials, i.e $\mathbb{P}_{1}(E) \subset$ $\mathcal{V}_{h}(E)$. It is immediate to verify that the dimension of $\mathbf{V}_{0, h}$ (the same happens for $\mathbf{V}_{h}$ ) equals the number of the interior vertices of the partition $\mathcal{T}_{h}$ plus those belonging to $\Gamma_{t}$, having fixed the values at vertices belonging to $\Gamma_{d}$ to incorporate Dirichlet boundary conditions. The construction of the global form $a_{h}\left(\rho_{h} ; \mathbf{u}_{h}, \mathbf{v}_{\mathbf{h}}\right)$ hinges upon the construction of local forms $a_{h}^{E}\left(\mathbf{u}_{h}, \mathbf{v}_{h}\right)$ : $\mathcal{V}_{h}(E) \times \mathcal{V}_{h}(E) \rightarrow \mathbb{R}$ approximating the integrals $\int_{E} \mathbf{C}_{0} \epsilon(\mathbf{u}): \epsilon(\mathbf{v}) d x$ so that

$$
a_{h}\left(\rho_{h} ; \mathbf{u}_{h}, \mathbf{v}_{h}\right)=\sum_{E \in \mathcal{T}_{h}} \rho_{E}^{p} a_{h}^{E}\left(\mathbf{u}_{h}, \mathbf{v}_{h}\right)
$$

where we employed the fact that $\left.\rho_{h}\right|_{E} \in \mathbb{P}_{0}(E)$.

It is important to remark that the the linear system stemming from (5) can be assembled employing only the values of $u_{h}$ and $v_{h}$ at the vertices of $\mathcal{T}_{h}$ (see [5] for more details) and the values of $\rho_{h}$ on each polygon. Using (6) it is immediate to see that, with a slight abuse of notation in denoting the vector of densities, the algebraic version of (5) reads as

$$
\mathbf{K}\left(\rho_{h}\right) \mathbf{U}=\sum_{E \in \mathcal{T}_{h}} \rho_{E}^{p} \mathbf{K}_{E}^{0} \mathbf{U}_{E}=\mathbf{F}
$$

where:

- $\rho_{h}$ is the vector of the element densities, whose components are $\rho_{E}$;

- $\mathbf{K}$ is the global stiffness matrix depending on $\rho_{h}$;

- $\mathbf{K}_{E}^{0}$ is the element stiffness matrix for the virgin material, i.e. associated to $a_{h}^{E}(\cdot, \cdot)$;

- $\mathbf{U}$ is the generalized displacement vector;

- $\mathbf{U}_{E}$ is the element displacement vector;

- $\mathbf{F}$ is the load vector.

Employing (5) and (7) the discrete version of the topology optimization problem (4) reads as follows

$$
\begin{cases}\min _{\rho_{\text {min }} \leq \rho_{E} \leq 1} & \mathcal{C}_{h}\left(\rho_{h}, \mathbf{u}_{h}\right):=\mathbf{U}^{T} \mathbf{K U}=\sum_{E \in \mathcal{T}_{h}} \rho_{E}^{p} \mathbf{U}_{e}^{T} \mathbf{K}_{e}^{0} \mathbf{U}_{e} \\ \text { s.t. } & \mathbf{K}(\mathbf{x}) \mathbf{U}=\mathbf{F}, \\ & \mathcal{W} / \mathcal{W}_{0} \leq V_{f},\end{cases}
$$

In the above equation, the objective function is the structural compliance $\mathcal{C}_{h}$, Eqn. $(8)_{2}$ enforces the discrete equilibrium equation discussed in Section 2.1 and Eqn. $(8)_{3}$ enforces the volume 
constraint. The weight $\mathcal{W}$ is computed multiplying the element density $\rho_{E}$ by the area of the polygonal elements of $\mathcal{T}_{h}$, while $\mathcal{W}_{0}$ stands for the volume of the whole design region. A lower bound $\rho_{\min }>0$ is enforced on each density unknown $\rho_{E}$ to avoid singularity of Eqn. 8$]_{2}$.

It must be remarked that the simple forms in Eqns. (7) and (8) are due to the adoption of piecewise constant discretization adopted for the density field.

\subsection{Mesh dependence and checkerboard numerical instabilities}

The Method of Moving Asymptotes (MMA) [24], which is an approach of mathematical programming, is herein adopted to iteratively solve the discrete problem in Eqn. (8). The algorithm searches the unknowns over the element-wise density discretization. It is well-known that this discrete scheme is affected by numerical instabilities, such as the arising of checkerboard patterns and mesh dependence, see e.g. [7]. Many strategies are available nowadays to solve both problems [22]. Following [19] and the various applications in stress-based optimal design, see e.g. [13], a density filter approach is herein adopted, instead of applying the filter to the objective function and its sensitivities, as done in most of cases. The original design variables $\rho_{E}$ are transformed in a new set of physical unknowns $\tilde{\rho}_{E}$ as:

$$
\tilde{\rho}_{E}=\frac{1}{\sum_{E^{\prime} \in \mathcal{T}_{h}} H_{E, E^{\prime}}} \sum_{E^{\prime} \in \mathcal{T}_{h}} H_{E, E^{\prime}} \rho_{E^{\prime}}, \quad H_{E, E^{\prime}}=\sum_{E^{\prime} \in \mathcal{T}_{h}} \max \left(0, r_{\text {min }}-\operatorname{dist}\left(E, E^{\prime}\right)\right) \text {. }
$$

In the above equation $\operatorname{dist}\left(E, E^{\prime}\right)$ is the distance between the centroids of the elements $E$ and $E^{\prime}$, whereas $r_{\text {min }}>d_{m}$ is the filter radius, $d_{m}$ being the square root of the area of each polygon in $\mathcal{T}_{h}$. The assumption $r_{\min }=1,5 d_{m}$ allows to avoid the arising of undesired checkerboard patterns. Larger values of $r_{\min }$ provide control on the minimum thickness of any member of the design.

\section{NUMERICAL SIMULATIONS}

A set of preliminary numerical simulations are presented in this section, adopting the proposed VEM procedure described above. Both structured and unstructured polygonal grids have been employed, see e.g. Figure 1 for an example of grids consisting of 501 elements.. A refer-
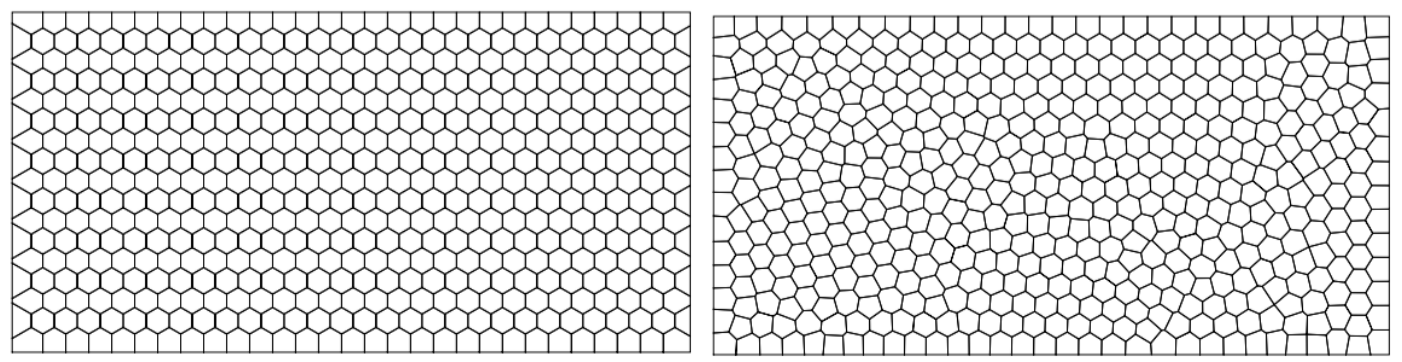

Figure 1: Examples of structured (left) and unstructured (right) polygonal grids consisting of 501 elements.

ence linear elastic isotropic material with Young modulus $E=1$ and Poisson's ratio $\nu=0.3$ is adopted. The volume fraction of available material is $V_{f}=0.3$ for the whole set of simulations. Moreover, to assess the proposed procedure with respect to well-known benchmark problems we consider different values of the filtering radius.

For the sake of comparison, in the following we compare our results with the analogous ones obtained by employing the classical bilinear displacement-based finite elements on Cartesian meshes, see [7]. 

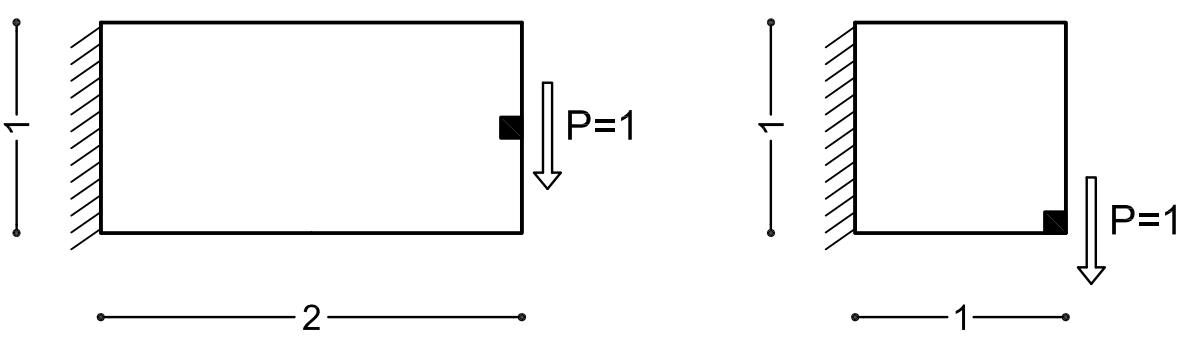

Figure 2: Geometry and boundary conditions for the numerical simulations: Example 1 (left) and Example 2 (right). In each case $P=1$ is the intensity of the unitary traction $\mathbf{f}_{t}$ applied as a nodal force oriented as indicated by the arrow.

\subsection{Example 1. A rectangular cantilever}

A first investigation is performed on the rectangular cantilever depicted in Figure 2(left). The reference solution is shown in Figure 3 (b) and has been obtained with bilinear displacementbased finite elements on a Cartesian grid consisting of 8192 squares $\left(2^{6}\right.$ elements lie along the thickness of the cantilever). The filter radius is $r_{\min }=3.0 d_{m}$. As expected, a truss-like structure arises where inclined members carry shear forces, whereas horizontal ones cope with bending actions. Figure 3 (a) shows the optimal design obtained by means of the VEM on a structured mesh consisting of 7990 polygonal elements $\left(2^{6}\right.$ elements lie along the thickness of the cantilever) and employing the same filter radius $r_{\text {min }}=3.0 d_{m}$. The achieved result is approximately the same found by the classical approach.

An additional set of simulations is performed adopting $2^{5}$ elements along the thickness of the cantilever, but preserving the same filter radius implemented in the previous investigations. Figure 4(a) shows the optimal design obtained with VEM on a structured mesh consisting of 2006 polygonal elements, whereas Figure 4(b) shows the optimal design achieved through the bilinear displacement-based approach for a regular mesh of 2048 square elements. Although the main layout of Figure 3 is recovered in both pictures, the displacement-based design provides an unexpected variation in the inclination of the thinner braces of the arising truss-like structure. The low available amount of material $\left(V_{f}=0.3\right)$ and the rough mesh of square elements make the optimizer find a 45-degree inclination, which means a local optimum instead of the expected global one. On the other hand, the VEM succeeds in finding the expected layout even in case of unstructured meshes, see the result shown in Figure 5 obtained on a mesh consisting of 2048 elements. This simulation employs the same filter radius $r_{\min }$ as above.

Finally, Figure 6 shows a comparison between the VEM approach (a) and the displacementbased one (b) for regular meshes of 32028 and 32768 elements, respectively ( $2^{7}$ elements lie along the thickness of the cantilever). Despite the adopted smaller filter radius $r_{\min }=1.5 d_{m}$, the achieved results are almost identical.

\subsection{Example 2. A square cantilever}

In the second example we consider the square cantilever depicted in Figure 2(right). Our VEM-based topology optimization method has been implemented employing unstructured grids of polygonal elements as those shown in Figure 1

First, an unstructured meshes with $2^{6}$ elements along the thickness of the cantilever is used. The overall number of elements is 4096 and the filter radius is $r_{\min }=1.5 d_{m}$ where, as before, $d_{m}$ is the square root of the average area of the polygonal elements in the unstructured grid. 


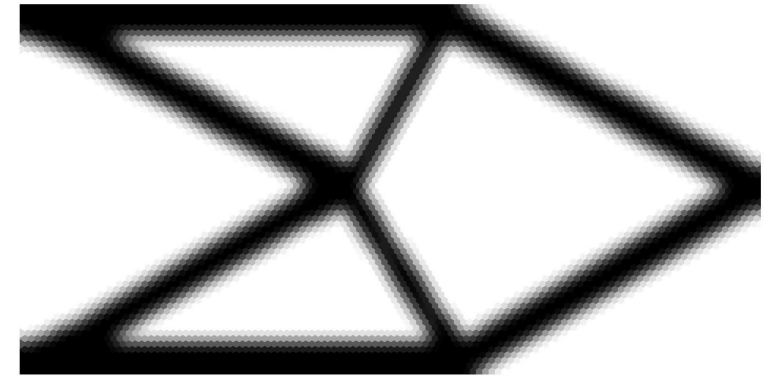

(a)

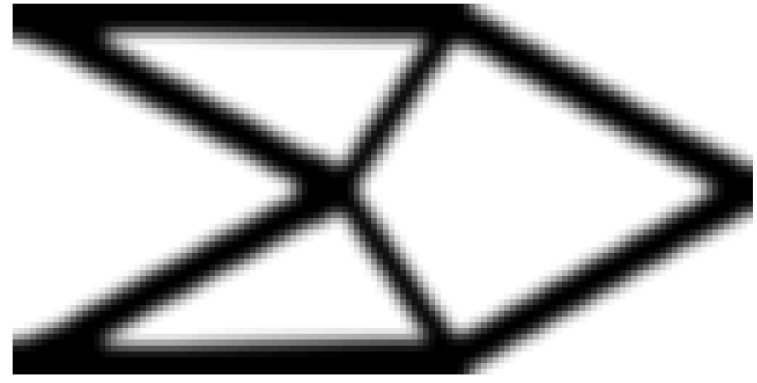

(b)

Figure 3: Example 1. Optimal topologies computed on on structured meshes with $2^{6}$ elements along the thickness of the cantilever: proposed VEM sed formulation (a), bilinear displacement-based formulation (b).

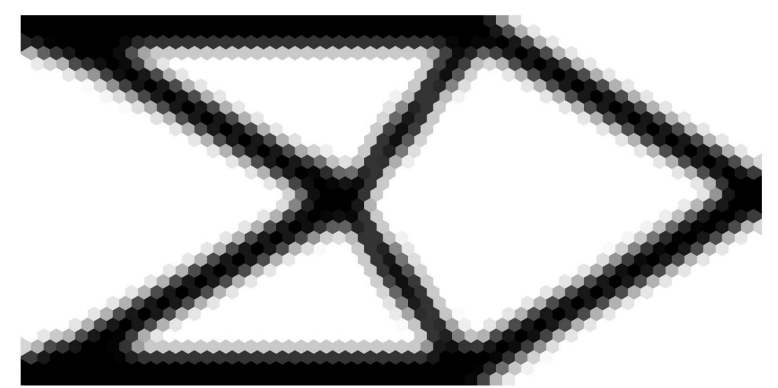

(a)

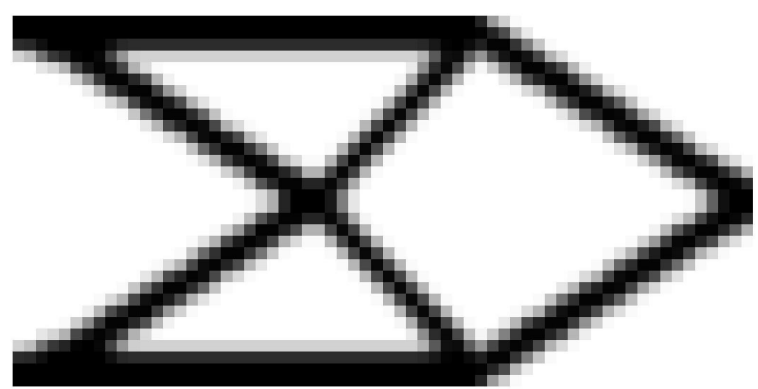

(b)

Figure 4: Example 1. Optimal topologies computed on structured meshes with $2^{5}$ elements along the thickness of the cantilever: proposed VEM formulation (a), bilinear displacement-based formulation (b).

The computed result is shown in Figure 7. As expected, a truss-like structure arises where the central node receives two major tensile-stressed bars and two minor compressive-stressed trusses. Figure 8 shows the computed optimal configuration for a stricter prescription on the filter radius, i.e. $r_{\min }=3.0 d_{m}$. The minimum member thickness enforced through the updated filter setup provides a simpler design with two ties and one big strut, in full agreement with the well-known solution of this benchmark problem, see [7]. Finally, in Figure 9 we show the optimal design computed when a finer mesh with $2^{7}$ elements along the thickness of the cantilever is employed. The overall number of polygonal elements is 16384 and the filter radius is $r_{\text {min }}=6.0 d_{m}$, that is approximately the same value used for the result presented in Figure 8. The same result is obtained, suggesting that no mesh dependence affects the proposed VEM formulation.

\section{CONCLUSIONS}

A set of preliminary numerical simulations has been presented to investigate the performance of the Virtual Element Method in driving a topology optimization algorithm. A classical volume-constrained minimum compliance problem has been tested, adopting an element-wise constant discretization for the unknown density field. Both structured and unstructured grids made of polygonal elements have been employed to solve two benchmark problems. For the sake of comparison, we have also presented the corresponding results obtained with bilinear displacement-based finite elements on Cartesian grids. Employing a sufficiently fine mesh, the same optimal layout is recovered by both methods. However, in case of a coarse mesh and 


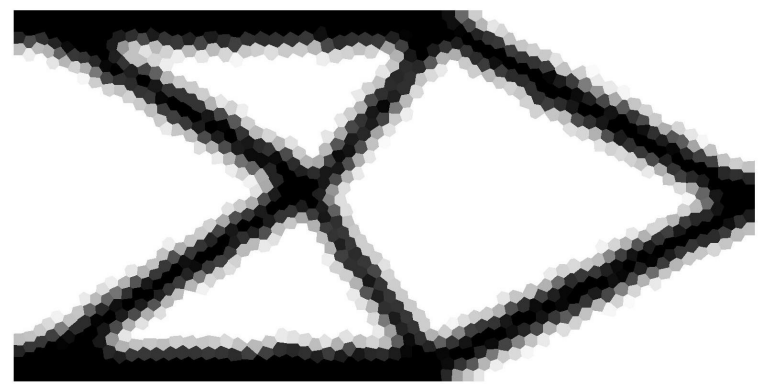

Figure 5: Example 1. Optimal topology achieved through the proposed VEM formulation for an unstructured mesh with $2^{5}$ elements along the thickness of the cantilever.

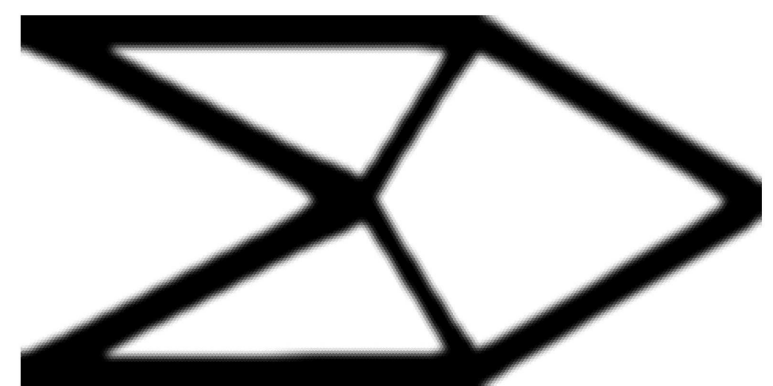

(a)

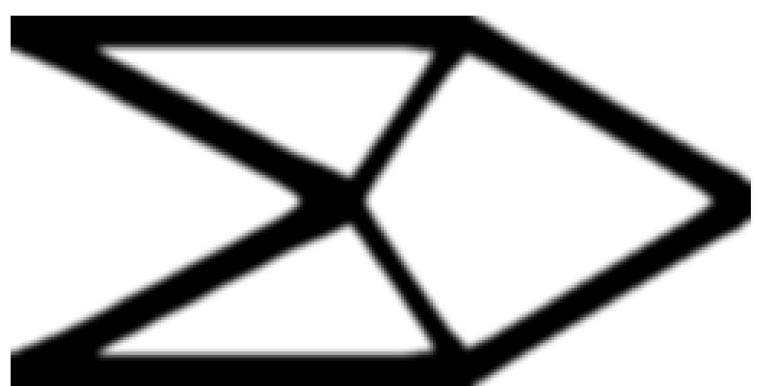

(b)

Figure 6: Example 1. Optimal topologies computed on structured meshes with $2^{7}$ elements along the thickness of the cantilever: proposed VEM formulation (a), bilinear displacement-based formulation (b).

low fraction of available material, the bilinear displacement-based approach is prone to convergence to a local optimum. Indeed, the orientation of the members is highly affected by the geometrical features of the underlying mesh of square elements. On the contrary, employing either structured or unstructured grids polygonal grids with approximately the same number of elements this issue is overcome and the expected global optimum is obtained (see Example 1). Additional numerical simulations assess that the proposed VEM seems to be fully mesh independent. Indeed, by using classical filtering techniques, the same layout is obtained independently of the number of mesh elements (see Example 2). The ongoing research is mainly concerned with investigations focusing on employing the VEM for the optimal design of incompressible materials thanks to the the full stability properties of the scheme for $\nu \rightarrow 0.5$.

\section{ACKNOWLEDGEMENTS}

Paola F. Antonietti has been partially supported by SIR Project n. RBSI14VT0S "PolyPDEs: Non-conforming polyhedral finite element methods for the approximation of partial differential equations" funded by MIUR. Marco Verani has been partially supported by GNCS-INDAM and by the Italian research grant Prin 2012 n. 2012HBLYE4 "Metodologie innovative nella modellistica differenziale numerica".

\section{REFERENCES}

[1] P. F. Antonietti, L. Beirão da Veiga, D. Mora, and M. Verani, A stream virtual element 


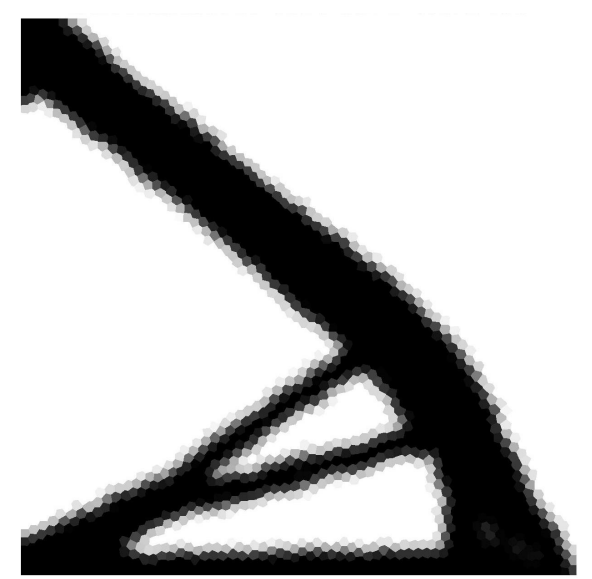

Figure 7: Example 2. Optimal topology computed with the proposed VEM formulation on an unstructured mesh with $2^{6}$ elements along the thickness of the cantilever and filter radius $r_{\min }=1.5 d_{m} \approx 1.5 / 2^{6}$ (left) and $r_{\min }=$ $3.0 d_{m} \approx 1.5 / 2^{5}$ (right).

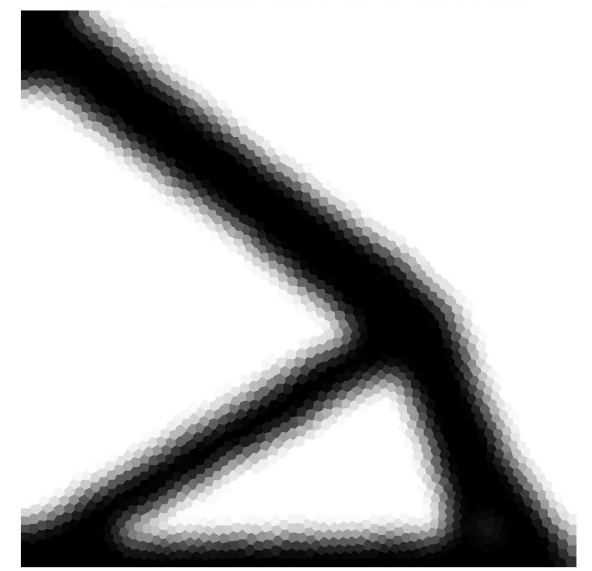

Figure 8: Example 2. Optimal topology computed with the proposed VEM formulation on an unstructured mesh with $2^{6}$ elements along the thickness of the cantilever and filter radius $r_{\min }=3.0 d_{m} \approx 1.5 / 2^{5}$.

formulation of the Stokes problem on polygonal meshes, SIAM Journal on Numerical Analysis, 52 (2014), 386-404.

[2] P. F. Antonietti, L. Beirão da Veiga, S. Scacchi, and M. Verani, A $C^{1}$ virtual element method for the Cahn-Hilliard equation with polygonal meshes, SIAM Journal on Numerical Analysis, doi:10.1137/15M1008117.

[3] L. Beirao da Veiga, F. Brezzi, A. Cangiani, G. Manzini, L. D. Marini, and A. Russo, Basic principles of virtual element methods, Mathematical Models and Methods in Applied Sciences, 23 (2103), 199-214.

[4] L. Beirão da Veiga, F. Brezzi, L. D. Marini, and A. Russo, The hitchhiker's guide to the virtual element method, Mathematical Models and Methods in Applied Sciences, 24 (2014), 1541-1573.

[5] L. Beirao da Veiga, F. Brezzi, and L. D. Marini, Virtual elements for linear elasticity problems SIAM Journal on Numerical Analysis, 51 (2013), 794-812. 


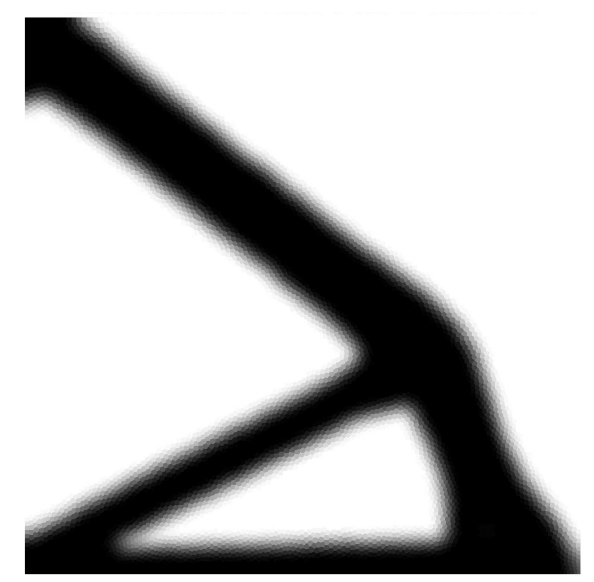

Figure 9: Example 2. Optimal topology computed with the proposed VEM formulation on an unstructured mesh with $2^{7}$ elements along the thickness of the cantilever and filter radius $r_{\min }=6.0 d_{m} \approx 1.5 / 2^{5}$.

[6] L. Beirão da Veiga and G. Manzini, A virtual element method with arbitrary regularity, IMA Journal of Numerical Analysis, 34 (2014), 759-781.

[7] M.P. Bendsøe, O. Sigmund, Topology optimization theory, methods and applications, New York, Springer, 2003.

[8] M.P. Bendsøe, Optimal shape design as a material distribution problem, Structural Optimization, 1 (1989), 193-202.

[9] M. F. Benedetto, S. Berrone, S. Pieraccini, and S. Scialò, The virtual element method for discrete fracture network simulations, Computer Methods in Applied Mechanics and Engineering, 280 (2014), 135-156.

[10] M. F. Benedetto, S. Berrone and S. Scialò, A globally conforming method for solving flow in discrete fracture networks using the Virtual Element Method, Finite Elements In Analysis and Design, 109 (2016), 23-36.

[11] F. Brezzi, R.S. Falk, and L.D. Marini, Basic principles of mixed Virtual Element Methods, ESAIM. Mathematical Modelling and Numerical Analysis, 48 (2014), 1227-1240.

[12] F. Brezzi and L. D. Marini, Virtual element methods for plate bending problems, Computer Methods in Applied Mechanics and Engineering, 253 (2013), 455-462.

[13] M. Bruggi, P. Duysinx, A stress-based approach to the optimal design of structures with unilateral behavior of material or supports, Structural and Multidisciplinary Optimization, 48 (2013), 311-326.

[14] M. Bruggi, P. Venini, Eigenvalue-based optimization of incompressible media using mixed finite elements with application to isolation devices, Computer Methods in Applied Mechanics and Engineering, 197 (2008), 1262-1279.

[15] M. Bruggi, M. Verani, A fully adaptive topology optimization algorithm with goaloriented error control, Computers and Structures, 89 (2011), 1481-1493. 
[16] A. Cangiani, G. Manzini, A. Russo, and N. Sukumar, Hourglass stabilization and the virtual element method, International Journal for Numerical Methods in Engineering, 102 (2015), 404-436.

[17] A.L. Gain, G.H. Paulino, L.S. Duarte, and I.F.M. Menezes, Topology optimization using polytopes virtual elements for linear elasticity problems, Computer Methods in Applied Mechanics and Engineering, 293 (2015), 411-430.

[18] A.L. Gain, C. Talischi, G.H. Paulino, On the Virtual Element Method for threedimensional linear elasticity problems on arbitrary polyhedral meshes, Computer Methods in Applied Mechanics and Engineering, 282 (2014), 132-160.

[19] C. Le, J. Norato, T.E. Bruns, C. Ha, D.D. Tortorelli, Stress-based topology optimization for continua,Structural and Multidisciplinary Optimization, 41 (2010), 605-620.

[20] G. Manzini, A. Russo, and N. Sukumar, New perspectives on polygonal and polyhedral finite element methods, Mathematical Models and Methods in Applied Sciences, 24 (2014), 1665-1699.

[21] D. Mora, G. Rivera, and R. Rodríguez, A virtual element method for the Steklov eigenvalue problem, Mathematical Models and Methods in Applied Sciences 25 (2015), 1421-1445.

[22] O. Sigmund O, J. Petersson, Numerical instabilities in topology optimization: a survey on procedures dealing with checkerboards, mesh-dependencies and local minima, Structural Optimization, 16 (1998), 68-75.

[23] O. Sigmund, P.M. Clausen, Topology optimization using a mixed formulation: an alternative way to solve pressure load problems, Computer Methods in Applied Mechanics and Engineering, 196 (2017), 1874-1889.

[24] K. Svanberg, Method of moving asymptotes - a new method for structural optimization, International Journal for Numerical Methods in Engineering, 24 (1897), 193-202.

[25] Y. Wang, Z. Kang, Q. He, Adaptive topology optimization with independent error control for separated displacement and density fields, Computers and Structures, 135 (2014), 5061. 